Università di Torino

elisabetta.rossi@unito.it

\title{
NOMI FEMMINILI NEI CORPORA ONLINE DI ANTICHI DOCUMENTI VOLGARI ITALIANI DEL MEDIOEVO
}

Parole chiave: TLIO, DATINI, corpora antroponimici, nomi medievali, varianti

Esistono ormai in rete molti strumenti che consentono l'accesso a documentazione volgare di epoca medievale e la ricerca si orienta soprattutto verso aspetti di tipo lessicale e linguistico. In particolare mi sono occupata di verificare quale può essere l'utilizzazione di queste fonti per una ricerca antroponimica, esaminando l'accessibilità dei diversi strumenti, il tipo di risultati ottenibili e inoltre la possibilità di estrarre dati e materiali mirati distinguendo le tipologie onomastiche: toponimi e antroponimi (maschili e femminili, primo nome, secondo nome, soprannome). Le mie ricognizioni hanno per il momento preso in considerazione due grandi corpora di italiano medievale reperibili in rete: TLIO (Tesoro della Lingua Italiana delle Origini) e DATINI (fondo di lettere di Francesco Datini). In particolare la mia ricerca si è focalizzata sui nomi femminili.

TLIO

Il Tesoro della Lingua Italiana delle Origini ${ }^{1}$ è un vocabolario storico dell'italiano antico, elaborato dai ricercatori e collaboratori dell'Opera del Vocabolario Italiano (OVI), Istituto del Consiglio Nazionale delle Ricerche con sede a Firenze, presso l'Accademia della Crusca.

Il corpus TLIO, la cui pubblicazione è cominciata nell'ottobre $1997^{2}$, contiene (al 14 luglio 2016, data dell'ultimo aggiornamento, sempre riportato sulla maschera iniziale) 2.117 testi per complessive 21.920 .635 occorrenze e 445.750 forme

\footnotetext{
${ }^{1}$ Consultabile all'indirizzo online http://www.ovi.cnr.it (oppure http://www.vocabolario.org, ultimo accesso il 30/10/2016), il TLIO copre l'arco temporale compreso tra le origini (il testo più antico è l'Indovinello veronese) e il 1375 (anno della morte di Boccaccio); di fatto sono compresi nel corpus anche testi che superano tale limite cronologico.

${ }^{2}$ Mosti 2010: 1-2.
} 
grafiche distinte. È reso disponibile in rete per le ricerche linguistiche e consente di scaricare brevi citazioni per uso di ricerca.

Le notizie contenute nell'introduzione al $\mathrm{TLIO}^{3}$ ci informano che la lemmatizzazione è sostanzialmente completa per quanto riguarda le forme grafiche, con un'esigua rimanenza di forme rimaste ancora non interpretate. Perciò la ricerca di un lemma consente di acquisire l'elenco completo delle forme del lemma stesso presenti nel corpus.

Ad oggi sono lemmatizzate 3.674 .650 occorrenze $^{4}$ (dati riferiti all'aggiornamento di luglio 2016), rinvenibili direttamente grazie alla ricerca per lemmi.

Le altre occorrenze delle forme trovate con la ricerca per lemmi sono reperibili selezionando anche l'opzione non lemmatizzati relativa al tipo di contesti. L'introduzione ribadisce, inoltre, che la lemmatizzazione del corpus dell'italiano antico è resa complessa dalla difficoltà che richiede l'interpretazione di numerosissimi contesti in molte varietà linguistiche diverse, in linguaggi diversi (poetico, giuridico, botanico, medico ecc.), e in testi editi con i più diversi criteri filologici, solo per una parte dei quali esistono strumenti di interpretazione (commenti, glossari ecc.) di qualità e affidabilità molto varia. Questo lavoro è stato iniziato fin dalle origini dell'Opera del Vocabolario, ed è passato attraverso metodi linguistici e strumenti informatici diversi e attraverso l'opera di molte persone. Per queste ragioni è possibile ancora incontrare nelle lemmatizzazioni presenti nel corpus incoerenze e anche veri e propri errori ${ }^{5}$, alla correzione dei quali si sta lavorando mentre si procede nel contempo ad ampliare il numero delle occorrenze lemmatizzate.

Il software lessicografico utilizzato dall'Istituto del CNR per elaborare e gestire TLIO è lo stesso usato per gli altri corpora di italiano antico: si chiama GATTO 6 , acronimo di Gestione degli Archivi Testuali del Tesoro delle Origini, ed è stato ideato da Domenico Iorio Fili presso l'Opera del Vocabolario Italiano. La versione web, denominata GATTOWEB, è interrogabile dall'ottobre $2005^{7}$.

Per estrarre dal software GATTO i lemmi interessanti per la ricerca antroponimica occorre impostare la categoria grammaticale n.p. (nomi propri) e il disambiguatore antr. (antroponimi, presenti in numero di $37810^{8}$ record, inclusi i casi

${ }^{3}$ Reperibili navigando oltre la maschera introduttiva del TLIO (online: http://tlioweb.ovi.cnr.it// HelpCorpora/Infotlio.html; ultimo accesso il 30/10/2016). L'attuale responsabile della lemmatizzazione è Elena Artale.

${ }^{4}$ Ibidem.

${ }^{5}$ Ibidem.

${ }^{6}$ Mosti 2010: 20.

${ }^{7}$ Indirizzo diretto (online: http://gattoweb.ovi.cnr.it, ultimo accesso il 30/10/2016), ma vi si accede anche dall'home page dell'OVI, cliccando il link "Interroga le banche dati" e poi l'icona relativa alla banca dati TLIO). Tale programma può essere usato utilizzando i principali programmi per la navigazione in rete.

${ }^{8}$ I numeri naturalmente sono in continuo aumento, dato il costante aggiornamento del corpus. 
dubbi $\left.^{9}\right)$, mentre per estrarre $\mathrm{i}$ toponimi è necessario fare uso del disambiguatore n.g. (nomi geografici, in numero di $21023^{10}$ ).

Vi sono poi casi in cui la colonna del disambiguatore non riporta alcuna indicazione, ad esempio in corrispondenza di nomi quali Durindana, Fulberta, Gioiosa, Altaclera, nomi propri delle spade dei cavalieri protagonisti dei poemi cavallereschi, cui, dunque, non è stata assegnata un'etichetta.

Per quanto riguarda gli antroponimi, non è possibile interrogare il sistema a livello più profondo, arrivando a distinguere primi, secondi nomi e nomi aggiunti. Per giungere a una disambiguazione certa in tal senso occorre cercare, tramite l'opzione "mostra contesti", uno per uno tutti i contesti testuali da cui sono estratti i nomi in questione.

Inoltre, provando ad eseguire tale operazione, appare subito evidente l'impossibilità di distinguere, oltre che tra primi, secondi nomi e nomi aggiunti, anche tra individui reali e nomi letterari, a meno di non vagliare ogni nome in ciascuna sua occorrenza tramite l'opzione "mostra contesti". Prendendo ad esempio un nome che può indifferentemente riferirsi a individui reali come a personaggi letterari, si può analizzare il caso di Elisabetta (Fig.1):

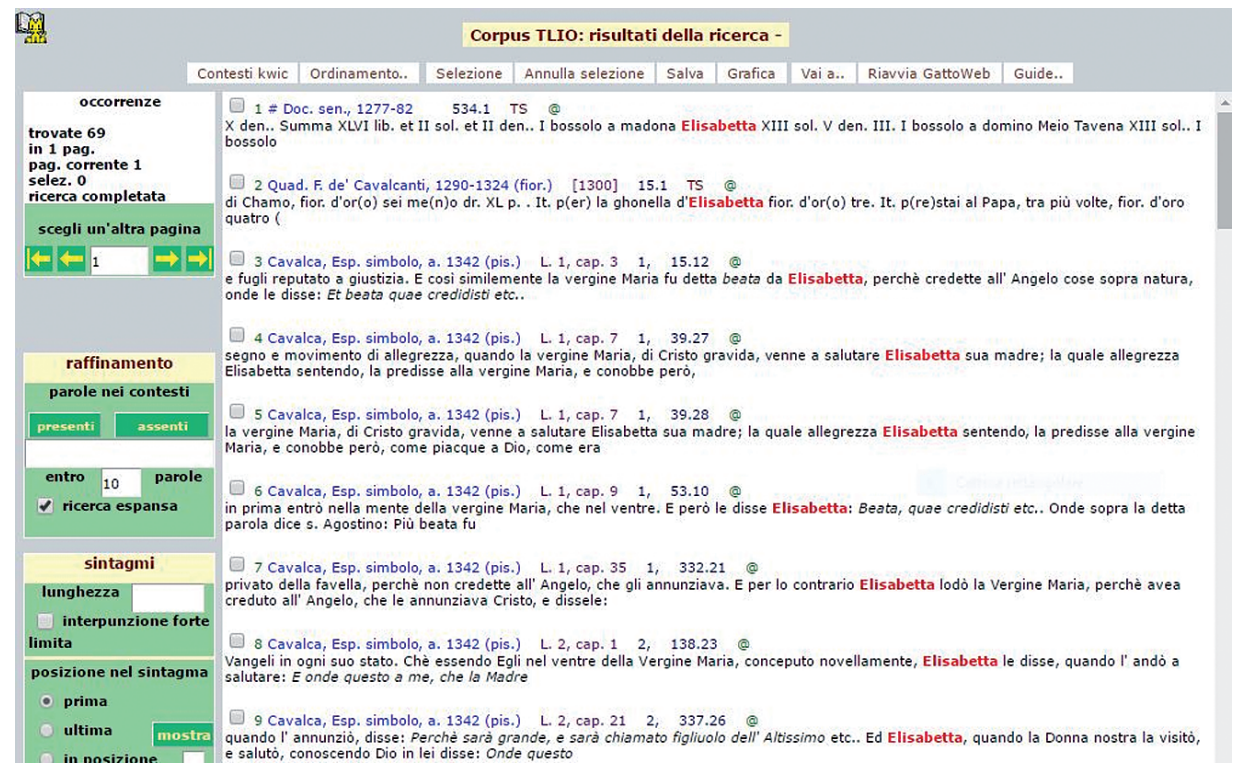

Fig. 1

${ }^{9}$ Vi sono un certo numero di antroponimi contrassegnati dal sistema con il disambiguatore antr?, casi ritenuti dubbi per diverse motivazioni (essenzialmente ambiguità o inconsuetudine), ad esempio Acquaviva e Scolpulzio.

${ }^{10} \mathrm{Vd}$. nota precedente. 
Per le occorrenze dalla 3 alla 9 il contesto di riferimento aiuta a ricostruire che il personaggio citato è la cugina della Vergine Maria e quindi ininfluente dal punto di vista di uno studio onomastico; per le prime due occorrenze, è necessario cliccare sulla rubrica (in blu) per aprire la scheda relativa all'opera di riferimento ed ipotizzare verosimilmente la presenza di individui reali:

\begin{tabular}{l}
\hline Dati bibliografici e statistici del testo - Google Chrome \\
$\square$ tlioweb.ovi.cnr.it/(S(v3cavu45 qcfned55tqvsdbuy))/CatSecond.aspx?calling=12\&sigla=1
\end{tabular}

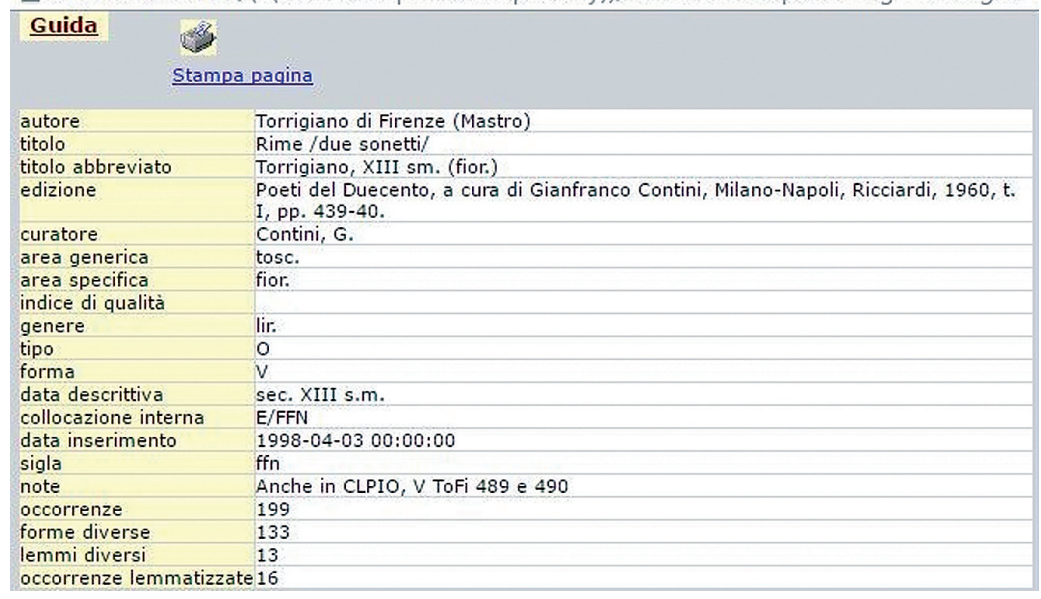

Fig. 2

\begin{tabular}{|c|c|c|}
\hline \multicolumn{2}{|c|}{ Dati bibliografici e statistici del testo - Google Chrome } & $\square$ \\
\hline \multicolumn{3}{|c|}{6 tlioweb.ovi.cnr.it/(S(2zyfow45pgljndvjhz03sj55))/CatSecond.aspx?calling $=128$ sigla $=m$} \\
\hline \multirow{2}{*}{\multicolumn{3}{|c|}{ Guida }} \\
\hline & & \\
\hline \multicolumn{3}{|c|}{ autore $\quad=$} \\
\hline titolo & \multicolumn{2}{|c|}{ Libro dell'entrata e dell'uscita di una Compagnia mercantile senese del secolo XIII } \\
\hline titolo abbreviato & \multicolumn{2}{|c|}{ Doc. sen., $1277-82$} \\
\hline edizione & \multicolumn{2}{|c|}{$\begin{array}{l}\text { Libro dell'entrata e dell'uscita di una Compagnia mercantile senese del secolo XIII, } \\
\text { a cura di Guido Astuti, Torino, Lattes, } 1934 \text {. }\end{array}$} \\
\hline curatore & \multicolumn{2}{|c|}{ Astuti, $\mathrm{G}$. } \\
\hline area generica & \multicolumn{2}{|l|}{ tosc. } \\
\hline area specifica & \multicolumn{2}{|l|}{ sen. } \\
\hline indice di qualità & \multicolumn{2}{|l|}{ TS } \\
\hline genere & \multicolumn{2}{|l|}{ doc. merc. } \\
\hline tipo & \multicolumn{2}{|l|}{0} \\
\hline forma & \multicolumn{2}{|l|}{ P } \\
\hline data descrittiva & \multicolumn{2}{|l|}{$1277-82$} \\
\hline collocazione interna & \multicolumn{2}{|l|}{ Cit/1. Libro.3 } \\
\hline data inserimento & \multicolumn{2}{|l|}{ 1997-04-21 00:00:00 } \\
\hline sigla & \multicolumn{2}{|l|}{$\mathrm{mk}$} \\
\hline \multicolumn{3}{|l|}{ note } \\
\hline occorrenze & \multicolumn{2}{|l|}{170921} \\
\hline forme diverse & \multicolumn{2}{|l|}{4261} \\
\hline lemmi diversi & \multicolumn{2}{|l|}{2382} \\
\hline occorrenze lemmatiz & 43086 & \\
\hline
\end{tabular}

Fig. 3 
Solo nel secondo caso (Fig. 3), giacché ci troviamo di fronte a una fonte documentaria (Il libro dell'entrata e dell'uscita di una compagnia mercantile senese del secolo XIII), abbiamo qualche elemento in più per ricostruire che possa trattarsi di un personaggio reale.

Ne consegue, dunque, che anche ricostruire il numero degli individui è difficile.

È possibile in alcuni casi uno studio di varianti e tuttavia non vi è una lemmatizzazione efficace che consenta una visione d'insieme di tali varianti: prendendo ad esempio il caso di un nome evidentemente solo letterario, Pentesilea, per trovare tutte le occorrenze relative al nome è necessario cercare separatamente le due forme Pantasilea (Fig. 4) e Pentesilea (Fig. 5):

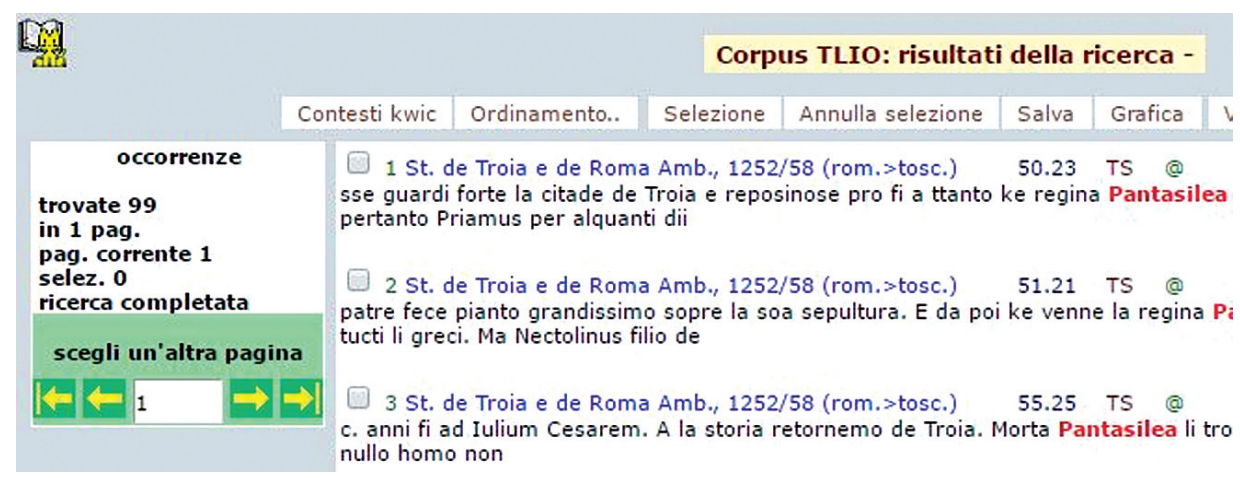

Fig. 4

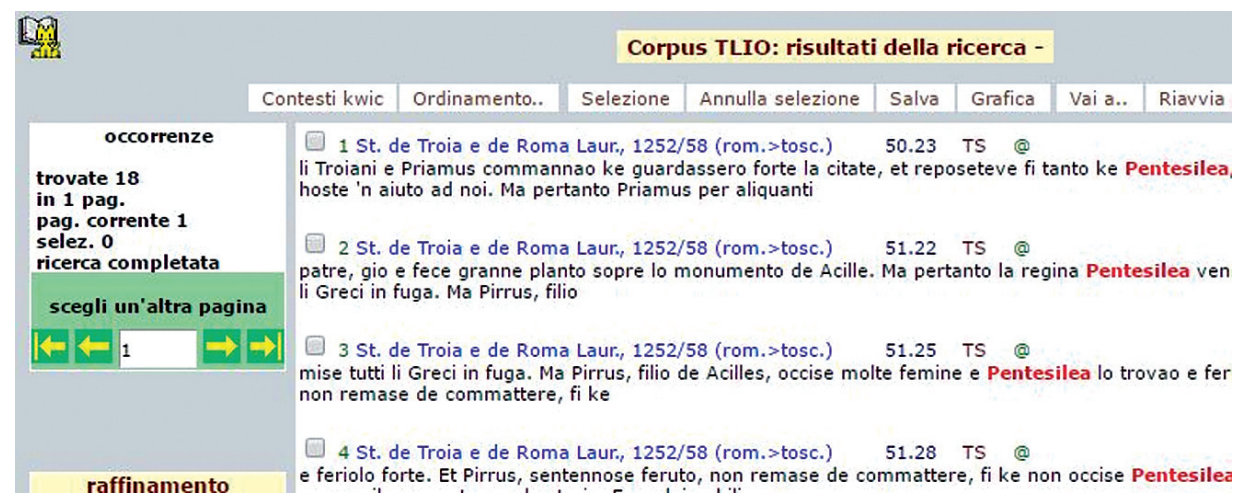

Fig. 5

A questo punto si può arrivare a dire che le 99 attestazioni di Pantasilea fanno di questa forma la maggioritaria rispetto a Pentesilea (18), anche se naturalmente, trattandosi di un personaggio letterario, non è possibile uno studio di tipo socioonomastico. 
In conclusione, il corpus TLIO non presenta ancora le caratteristiche per essere una fonte di utilizzo agevole per lo studio antroponimico, soprattutto per l'assenza di mappe distintive e di lemmatizzazioni efficaci.

\section{DATINI}

DATINI è il corpus lemmatizzato delle lettere e dei documenti editi dell'Archivio DATINI, fondo conservato nell'Archivio di Stato di Prato, fonte di grandissimo interesse per la ricostruzione della storia del mondo mercantile europeo nella seconda metà del Trecento. L'archivio DATINI contiene oltre 150.000 documenti sciolti e circa 600 registri $^{11}$. Nel 2003 l'Archivio di Stato di Prato affidava all'Opera del Vocabolario Italiano l'allestimento di una banca dati informatizzata contenente le lettere e i documenti editi dell'archivio DATINI ${ }^{12}$, con la richiesta di indicizzare i nomi di persona e di luogo e di lemmatizzare i termini appartenenti ad alcuni settori di lessico: quello storico-economico, commerciale (incluso il commercio marittimo), militare e storico-giuridico, nonché i tecnicismi e le date rilevanti. Una seconda richiesta verteva sulla possibilità di raggruppare $\mathrm{i}$ lemmi prodotti in base ai rispettivi ambiti tematici di afferenza, e ottenere un livello di ricerca che guidasse l'utente nell'interrogazione del corpus. È nato così il corpus lemmatizzato del carteggio Datini, allestito con lo stesso programma che gestisce il corpus TLIO dell'OVI, il Gatto, in una versione appositamente dedicata ${ }^{13}$.

Il corpus consta di 2.511 testi e 45.259 forme, per un totale di 977.034 occorrenze, di cui 126.663 lemmatizzate, con 6.510 lemmi e 22 iperlemmi (l'iperlemma è ciascuna delle 22 categorie entro le quali sono stati distribuiti i lemmi del corpus ad esempio abbigliamento, arredi, alimenti, etc., ma sono solo lemmatizzati e non iperlemmatizzati antroponimi, toponimi, termini e verbi afferenti al campo religioso o all'agricoltura, le parti del corpo, i nomi della settimana e i termini generici).

L'intera documentazione del corpus abbraccia un intervallo temporale che va dal 1367 al $1432^{14}$ e contiene, oltre alle lettere e ai documenti commerciali delle varie filiali della compagnia Datini sparse per la penisola e fuori dall'Italia (adAvignone enell'allora Regno di Aragona), il testamento di Francesco Datini e gli scambi epistolari in cui convivono la dimensione pubblica e i rapporti privati tra gli scriventi: il carteggio di Francesco con la moglie Margherita Bandini, quello con l'amico e notaio Lapo Mazzei e quello con il medico Naddino.

Significative sono la vastità geografica rappresentata dal corpus datiniano, nonchè il notevole assortimento linguistico, ricco di varietà italoromanze e non: 2408 testi

\footnotetext{
${ }^{11}$ Informazioni tratte da http://datini.archiviodistato.prato.it/ (ultimo accesso il 30/10/2016).

12 Artale 2006.

13 Ibidem.

${ }^{14}$ Ibidem: 2.
} 
rappresentano il toscano (nelle sue varianti e nelle peculiari realizzazioni di scriventi non toscani o da lungo tempo residenti fuori dalla Toscana), 38 il marchigiano, 14 il siciliano, 8 il campano, 2 il veneto, un testo rispettivamente il calabrese, il laziale e il ligure; a questi vanno aggiunti 28 testi in latino, 9 in catalano e uno in tedesco ${ }^{15}$.

La marcatura dei lemmi è stata effettuata utilizzando le seguenti categorie ${ }^{16}$ :

agg. - aggettivo

antr. - antroponimo

avv. - avverbio

n.g. - nome geografico (nome di luogo)

n.p. - nome proprio

s.f. (eventualmente s.f.pl.) — sostantivo femminile (sostantivo femminile plurale)

s.i. - sostantivo di genere incerto

s.m. (eventualmente s.m.pl.) - sostantivo maschile (sostantivo maschile plurale)

v. - verbo

In DATINI, una volta selezionata la categoria n.p., è possibile estrarre tutte le voci che sono state lemmatizzate con categoria lessicale antr. tutti i nomi di persona, eventuali soprannomi, e cariche specifiche nel caso in cui l'indicazione rimandi ad un preciso personaggio storico $^{17}$.

Data la grande variazione grafica nei nomi propri, sono state effettuate alcune normalizzazioni grafiche, di cui l'introduzione al corpus fornisce qualche esempio:

1) le forme niccolaio, niccolo, nicolò, sono state ricondotte al lemma niccolò; le forme niccola, nicola, sono state ricondotte al lemma nicola;

2) l'alternanza buo-/ bonsignore è stata ricondotta al lemma senza dittongo bonsignore;

3) le forme del tipo gabriello sono state ricondotte al lemma gabriele;

4) le forme del tipo ghirig- / greg- / grigoro (-orio) sono state ricondotte al lemma gregorio;

5) le forme del tipo guasparre(-o) sono state ricondotte a gaspare;

6) le forme del tipo ludovic(-a) sono state ricondotte a lodovico(-a);

7) nei lemmi è stata sempre adoperata la grafia iacopo (e non jacopo).

L'introduzione al corpus fornisce anche interessanti informazioni in merito al trattamento degli antroponimi femminili, che, si dice, sono stati trattati, quando

15 Ibidem

${ }^{16}$ Le informazioni che seguono sono tratte dalle istruzioni infocorpus, reperibili navigando oltre la maschera introduttiva di DATINI, all'indirizzo (http://aspweb.ovi.cnr.it/(S(mwqipyavxrpipynbklbatfzj))/ HelpCorpora/Infoasp.html; ultimo accesso il 30/10/2016).

${ }^{17}$ L'iniziale minuscola anche nei nomi propri è dovuta al software GATTO (che non distingue tra maiuscole e minuscole). Per aderenza al software, nelle citazioni dirette dal corpus ho mantenuto l'iniziale minuscola. 
possibile, come i maschili (es. lisa di ugolotto degli agli); solo in mancanza del cognome e/o del patronimico sono state date indicazioni del tipo donna di..., vedova di..., madre di..., serva di... o il semplice monna (o madonna) — riportando il titolo dal documento - posposti al nome, tra parentesi (es. diana, donna di iacopo zarini). Se è noto il nome della madre si è utilizzato un lemma del tipo sandra (figlia di leonarda panciatichi).

Nel caso di ginevra di francesco datini, si è preferito lemmatizzare ginevra (figlia naturale di francesco datini) piuttosto che ginevra di francesco datini.

La categoria lessicale antr. è stata usata anche per i nomi di famiglia, segnalati come tali nel disambiguatore.

Sono stati lemmatizzati come n.g. (nome geografico) i nomi di città, paesi, contrade, località, vie, piazze, porte; sono stati lemmatizzati come n.g. anche specifici edifici, come chiese, monasteri, palazzi, ospedali.

Vi sono, inoltre, specifici disambiguatori appositamente studiati per marcare i nomi di nave (nome di nave) e i nomi di ordini religiosi (ordine religioso).

Le forme antroponimiche estratte selezionando la categoria grammaticale n.p. e filtrate dalla categoria lessicale antr. sono in tutto 6.139; i toponimi sono 60, i nomi di nave 34 e i nomi di ordini religiosi 14.

Gli antroponimi sono stati raccolti per stringa onomastica dell'individuo (diana donna di iacopo zarini) e si intuisce, dunque, la necessità di lemmatizzare sulla forma normalizzata, dopo averla estratta dalla stringa onomastica.

\section{NOMI FEMMINILI IN DATINI}

I primi nomi femminili, estratti da DATINI vagliando gli antroponimi registrati, sono in tutto 245, inclusi i casi dubbi. Questi 245 antroponimi sono riconducibili a 79 forme normalizzate (il numero può essere soggetto, come vedremo, a lievi oscillazioni per via dei criteri di lemmatizzazione utilizzati), a cui sono collegate le numerose varianti; poiché ciascun nome può essere riferito più volte alla stessa persona va precisato anche il numero degli individui, che ammonta a 149, inclusi i casi dubbi.

Tra i vantaggi vi è senz'altro il fatto che il corpus sia reale: gli individui citati sono persone realmente esistite e quindi databili. Inoltre, sono significative la visibilità sugli identificatori sociali (madonna o serva, ad esempio) e la ricostruzione dell'identità dei personaggi, nonché l'ampia variabilità grafico-fonetica, che permette di studiare il corpus dal punto di vista linguistico.

Tra le criticità si evidenzia il fatto che il sistema per ricavare i nomi non sia immediato e numerose operazioni siano necessarie per rendere il corpus utilizzabile dal punto di vista onomastico: prevede innanzitutto delle marcature di genere e lo scorporo dei dati della stringa onomastica, che risulta aggregata (Fig. 6): 


\begin{tabular}{|c|c|c|c|}
\hline lemma & cat less - & forma & disambiguatore \\
\hline francesca (vedova di ticcio) & antr. & franciescha & \\
\hline francesca di domenico bandini & antr. & fancescha & \\
\hline francesca di domenico bandini & antr. & fraciescha & \\
\hline francesca di domenico bandini & antr. & francesca & \\
\hline francesca di domenico bandini & antr. & francescha & \\
\hline francesca di domenico bandini & antr. & franciescha & \\
\hline francesca di francesco & antr. & franciescha di franciescho & \\
\hline francesca di giusto (monna) & antr. & franciescha di gusto & \\
\hline gaia (donna di piero di giunta del rosso) & antr. & gaia & \\
\hline gaia (donna di piero di giunta del rosso) & antr. & ghaia & \\
\hline gaia (monna) & antr. & ghaia & donna di piero di giunta del $r$ \\
\hline gemma & antr. & gemma & monna gemma? \\
\hline gemma (monna) & antr. & giema & \\
\hline ghita (donna di matteo ghetto) & antr. & ghita & \\
\hline ghita (donna di matteo ghetto) & antr. & gita & \\
\hline ghita di geri & antr. & gita di gieri & \\
\hline gianna (madre di baldello d'a. bovattieri) & antr. & giana & \\
\hline ginevra (figlia naturale di francesco datini) & antr. & g. di francesco & \\
\hline ginevra (figlia naturale di francesco datini) & antr. & ginevera & \\
\hline ginevra (figlia naturale di francesco datini) & antr. & ginevra & \\
\hline ginevra (figlia naturale di francesco datini) & antr. & zinevra & \\
\hline giovanna (monna) & antr. & giovana & \\
\hline giovanna (monna) & antr. & giovanna & \\
\hline giovanna (serva di margherita di domenico bandini) & antr. & giovana & \\
\hline
\end{tabular}

Fig. 6

Solo la ricostruzione di un database mirato può, dunque, rendere funzionale e agevole la consultazione del corpus.

Vi sono forme che si presentano in un'unica occorrenza: è indicata la variante attestata, qualora non coincida con quella base di lemmatizzazione:

Tabella 1

\begin{tabular}{|c|l|l|}
\hline \multicolumn{3}{|c|}{ Nomi a una occorrenza } \\
\hline ID & \multicolumn{1}{|c|}{ Nome } & Forma attestata \\
\hline 1. & Anna & {$\left[\right.$ Beloza $^{18}$} \\
\hline 2. & Bellozza & \\
\hline 3. & Benedetta & \\
\hline 4. & Benvenuta & \\
\hline 5. & Bionda & Cholomba \\
\hline 6. & Boneca & \\
\hline 7. & Colomba & \\
\hline 8. & Dialta & \\
\hline 9. & Dolce & Dora \\
\hline 10. &
\end{tabular}

\footnotetext{
${ }^{18}$ Nome non ricavabile dall'elenco.
} 


\begin{tabular}{|c|c|c|}
\hline \multicolumn{3}{|c|}{ Nomi a una occorrenza } \\
\hline ID & Nome & Forma attestata \\
\hline 11. & Eva & \\
\hline 12. & Farillera & \\
\hline 13. & Fattorina & \\
\hline 14. & Filippa & Filipa \\
\hline 15. & Gianna & Giana \\
\hline 16. & Gostanza & Gostança \\
\hline 17. & Gramigna & Ghramignia \\
\hline 18. & Jacopa & \\
\hline 19. & Lodola & \\
\hline 20. & Lorenza & \\
\hline 21. & Lorita & \\
\hline 22. & Luca & Lucha \\
\hline 23. & Maddalena & Madalena \\
\hline 24. & Masa & \\
\hline 25. & Mattea & \\
\hline 26. & $\mathrm{Mea}$ & \\
\hline 27. & Mina & \\
\hline 28. & Orsetta & Orseta \\
\hline 29. & Orta & \\
\hline 30. & Papera & \\
\hline 31. & Petrazza & Petraza \\
\hline 32. & Picchina & \\
\hline 33. & Sandra & \\
\hline 34. & Severina & \\
\hline 35. & Sibilla $^{19}$ & Reina \\
\hline 36. & Spina & \\
\hline 37. & Tessa & \\
\hline 38. & Tonina & \\
\hline 39. & Vivola & \\
\hline 40. & Zita & \\
\hline
\end{tabular}

${ }^{19}$ Nome presente in elenco ma non attestato. 
Vi sono due nomi che mettono in luce ulteriori criticità del sistema di lemmatizzazione: infatti, uno dei nomi presenti nell'elenco estratto dal corpus, Sibilla, non è realmente attestato, poiché la forma presente nel testo non è Sibilla, ma Reina: l'appellativo è riferito a Sibilla de Fortià, regina d'Aragona, e ciò ha spinto i compilatori a lemmatizzare (arbitrariamente) sul primo nome del personaggio, la cui identità è ricostruita con certezza, pur, dunque, in assenza del nome. Opposto il caso di Bellozza (presente nella variante Beloza), nome assente nell'elenco estraibile dal corpus e da me aggiunto: questo nome è stato ricavato dalle varianti di Bartolomea (lemmatizzata, infatti, come Bartolomea detta Bellozza); anche qui si ravvisa, quindi, un'arbitrarietà nel lemmatizzare sulla base di un nome in realtà assente dal testo. Casi analoghi si vedranno anche tra i nomi che contano più di un'attestazione.

Propongo ora un elenco dei nomi più attestati per frequenza decrescente, con riferimento al tipo onomastico; accanto è riportato il numero di individui nelle diverse forme e le relative attestazioni:

Tabella 2

\begin{tabular}{|c|c|c|c|c|c|}
\hline ID & Nome & $\begin{array}{c}\text { Individui } \\
\text { per tipo } \\
\text { onomastico }\end{array}$ & $\begin{array}{l}\text { Numero } \\
\text { individui } \\
\text { per forma }\end{array}$ & $\begin{array}{l}\text { Numero } \\
\text { attestazioni } \\
\text { per forma }\end{array}$ & Note \\
\hline 1. & Caterina, Tina & 13 & $11 ; 2$ & $16 ; 4$ & \\
\hline 2. & $\begin{array}{l}\text { Giovanna; Nanna; } \\
\text { Vanna }\end{array}$ & 12 & $6,5,1$ & $14 ; 9 ; 2$ & \\
\hline 3. & $\begin{array}{l}\text { Margherita Ghita, } \\
\text { Chita }\end{array}$ & 8 & $5 ; 2 ; 1$ & $19 ; 3 ; 3$ & $\begin{array}{l}\text { Lemmatizzati sotto } \\
\text { Margherita anche Madama } \\
\text { e Reina }\end{array}$ \\
\hline 4. & Bartolomea & 8 & $7 ; 1$ & $10 ; 3$ & $\begin{array}{l}\text { Lemmatizzato sotto } \\
\text { Bartolomea anche Bellozza }\end{array}$ \\
\hline 5. & Francesca; Checca & 7 & $6 ; 1 ?$ & $12 ; 2$ & $\begin{array}{l}\text { Non ci sono specifiche su } \\
\text { Checca }\end{array}$ \\
\hline 6. & Antonia & 5 & 5 & 8 & \\
\hline 7. & Beatrice, Bice & 4 & $2 ; 2$ & $2 ; 2$ & \\
\hline 8. & Piera & 4 & 4 & 5 & \\
\hline 9. & Agnola & 3 & 3 & 5 & \\
\hline 10. & Lisa & 3 & 3 & 4 & \\
\hline 11. & Andrea & 3 & 3 & 3 & \\
\hline 12. & Lapa & 3 & 3 & 3 & \\
\hline 13. & Chiara & 2 & 2 & 3 & $\begin{array}{l}\text { Lemmatizzata sotto Chiara } \\
\text { anche Monaca }\end{array}$ \\
\hline 14. & Leonarda & 2 & 2 & 3 & \\
\hline
\end{tabular}




\begin{tabular}{|c|c|c|c|c|c|}
\hline ID & Nome & $\begin{array}{c}\text { Individui } \\
\text { per tipo } \\
\text { onomastico }\end{array}$ & $\begin{array}{l}\text { Numero } \\
\text { individui } \\
\text { per forma }\end{array}$ & $\begin{array}{c}\text { Numero } \\
\text { attestazioni } \\
\text { per forma }\end{array}$ & Note \\
\hline 15. & Lucia & 2 & 2 & 3 & \\
\hline 16. & Niccolosa & 2 & 2 & 3 & \\
\hline 17. & Diana & 2 & 2 & 2 & \\
\hline 18. & $\begin{array}{l}\text { Domenica, } \\
\text { Domenichina }\end{array}$ & 2 & $4 ; 1$ & 5 & \\
\hline 19. & Fiore & 2 & 2 & 2 & \\
\hline 20. & Lena & 2 & 2 & 2 & \\
\hline 21. & Rosa & 2 & 2 & 2 & \\
\hline 22. & Giuliva & 1 & 1 & 5 & \\
\hline 23. & Chiaramonda & 1 & 1 & 4 & \\
\hline 24. & Cilia & 1 & 1 & 4 & \\
\hline 25. & Ginevra & 1 & 1 & 4 & \\
\hline 26. & Gregoria & 1 & 1 & 4 & \\
\hline 27. & Dianora & 1 & 1 & 3 & \\
\hline 28. & Gaia & 1 & 1 & 3 & \\
\hline 29. & Isabetta & 1 & 1 & 3 & \\
\hline 30. & Regina & 1 & 1 & 3 & $\begin{array}{l}\text { Lemmatizzata sotto Regina } \\
\text { anche Madonna }\end{array}$ \\
\hline 31. & Tinga & 1 & $1 ?$ & 3 & \\
\hline 32. & Beldì & 1 & 1 & 2 & \\
\hline 33. & Gemma & 1 & 1 & 2 & \\
\hline 34. & Paola & 1 & 1 & 2 & $\begin{array}{l}\text { Lemmatizzata sotto Paola } \\
\text { anche Coverciana }\end{array}$ \\
\hline 35. & Parta & 1 & 1 & 2 & \\
\hline 36. & Salvestra & 1 & 1 & 2 & \\
\hline 37. & Simona & 1 & 1 & 2 & \\
\hline 38. & Taddea & 1 & 1 & 2 & \\
\hline 39. & Villana & 1 & 1 & 2 & \\
\hline
\end{tabular}

Un caso interessante riguarda Margherita: come nel caso precedentemente citato di Sibilla, anche qui troviamo due appellativi (Reina e Madama) lemmatizzati come Margherita in quanto riferiti a Margherita di Durazzo (attestata con il suo nome una sola volta). 
Simile il caso relativo a Chiara: due delle tre attestazioni presenti sono ricondotte al personaggio di Chiara Gambacorti, ma in uno solo dei casi la donna è citata come Chiara, infatti la seconda attestazione è in realtà l'appellativo Monaca, chiaro riferimento alla sua condizione di monacazione; anche qui Monaca è stato lemmatizzato come Chiara.

Degno di nota anche il caso di Regina, presente in tre attestazioni (tutte riferite a Regina della Scala) lemmatizzate sotto Regina sebbene una delle occorrenze, ancorché riferita alla sua persona, sia l'appellativo Madonna.

Si può citare ancora il caso di Paola, in due attestazioni riferite al medesimo personaggio (citata in elenco come paola detta la coverciana), è attestata una volta come Paola (variante Paghola) e un'altra come Coverciana, elemento di chiaro carattere soprannominale. Occorre dunque vagliare una a una le forme effettivamente attestate per indagarne la significatività onomastica, poiché, come si è dimostrato, spesso l'etichettatura utilizzata in sede di lemmatizzazione rischia di celare forme che non sono semplici varianti grafiche o fonetiche e ciò può essere fuorviante per il linguista.

Tina è ipocoristico di Caterina, come conferma la documentazione storica; interessante per gli alterati Tinuca e Tinuccia, correttamente lemmatizzati sotto Tina.

Tabella 3

\begin{tabular}{|l|l|l|l|}
\hline \multicolumn{1}{|c|}{ Elemento 1} & \multicolumn{1}{c|}{ Lemma } & \multicolumn{1}{c|}{ Cat less } & \multicolumn{1}{c|}{ Forma } \\
\hline Tina & Tina & antr. & tina \\
\hline Tina & tina di niccolò dell'ammannato tecchini & antr. & tina \\
\hline Tina & tina di niccolò dell'ammannato tecchini & antr. & tinuca \\
\hline Tina & tina di niccolò dell'ammannato tecchini & antr. & tinuccia \\
\hline
\end{tabular}

Un procedimento evidentemente non omogeneo, quello di lemmatizzare gli alterati sotto la forma base: infatti nel caso di Domenica, l'alterato Domenichina (nella variante Domeneghina) è lemmatizzato a parte (e nel mio elenco è invece contata tra le varianti di Domenica, per omogeneità).

Tabella 4

\begin{tabular}{|l|l|l|l|}
\hline \multicolumn{1}{|c|}{ Elemento 1 } & \multicolumn{1}{|c|}{ Lemma } & Cat less & \multicolumn{1}{|c|}{ Forma } \\
\hline Domenica & domenica (donna di meo di simone) & antr. & domenica \\
\hline Domenica & domenica (donna di meo di simone) & antr. & domenicha \\
\hline Domenica & domenica (donna di meo di simone) & antr. & domeniche \\
\hline Domenica & domenica (donna di meo di simone) & antr. & domina \\
\hline Domenichina & domenichina (donna di piero di giusto benintendi) & antr. & domeneghina \\
\hline
\end{tabular}


A parte andrebbe considerata la forma Domina per Domenica: potrebbe trattarsi di un soprannome, bisognerebbe indagare consultando il testo originale nel suo contesto.

Complessa è la valutazione di Tigna, che potrebbe essere una forma soprannominale anche maschile ${ }^{20}$, come testimonia Brattö, che cita Tigna come forma da confrontare con Tignosus, indicato come soprannome e presente in quattro attestazioni tra i nomi meno frequenti del Libro di Montaperti; è confrontabile, inoltre, con un personaggio maschile denominato il Tigna, presente nella commedia seicentesca Il forca di Niccolò Amenta ${ }^{21}$.

Tabella 5

\begin{tabular}{|c|c|c|c|}
\hline Elemento 1 & Lemma & Cat less & Forma \\
\hline Tigna & tigna & antr. & tingna \\
\hline
\end{tabular}

Certamente femminile è la forma Tinga (come precisato dalla fonte). Non devono stupire le oscillazioni grafiche -ng-, -ngn- e -gn-, comuni nella tradizione manoscritta per rappresentare la $-n$ - palatale:

Tabella 6

\begin{tabular}{|c|c|c|c|}
\hline Elemento 1 & Lemma & Cat less & Forma \\
\hline Tinga & tinga (monna) & antr. & tinga \\
\hline Tinga & tinga (monna) & antr. & tingha \\
\hline
\end{tabular}

I casi dubbi sono tali a causa dell'assenza di ulteriori informazioni circa gli individui citati: ad esempio il due volte attestato Checca non ha riferimenti, per cui le due attestazioni potrebbero riferirsi alla stessa persona come a due differenti; tuttavia è maggiormente presumibile che si tratti di uno solo, giacché in presenza di tratti di disambiguazione i compilatori del corpus, come hanno fatto altrove, avrebbero specificato la presenza di due personaggi.

Propongo ora un grafico relativo alle attestazioni dei nomi pluriattestati, per numero di individui (Fig. 7).

\footnotetext{
20 Brattö 1955: 208

21 Amenta 1753.
} 

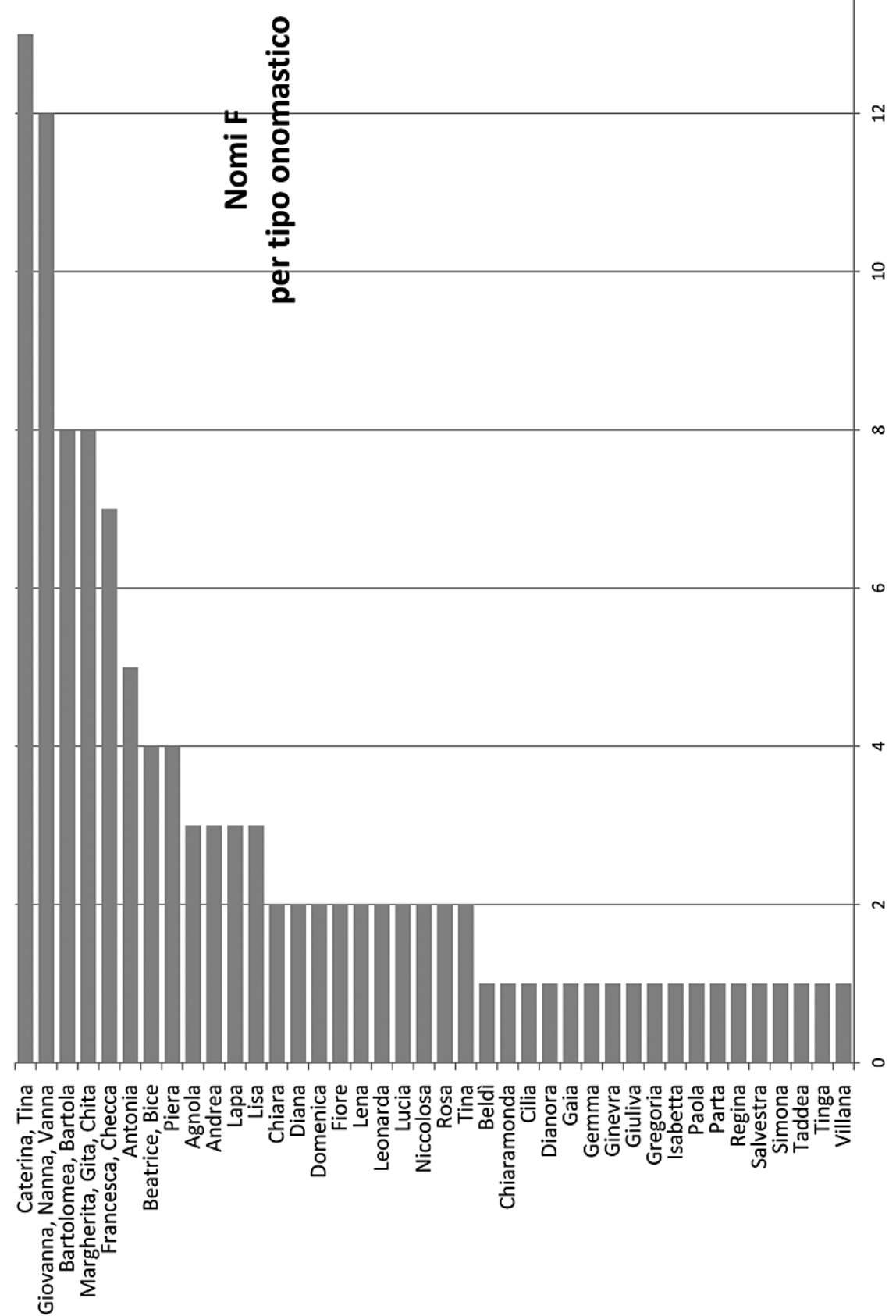
I tipi onomastici più rappresentati sono:

Tabella 7

Caterina (13 individui totali, di cui 11 nella forma a lemma), sostenuto da Tina, ipocoristico $^{22}$

Giovanna (12 individui totali, di cui 6 nella forma a lemma), sostenuto dagli ipocoristici Nanna (5) e Vanna (1)

$12 \quad 6 ; 5 ; 1$

a seguire

\begin{tabular}{|l|c|c|}
\hline $\begin{array}{l}\text { Margherita (8 individui totali, di cui } 5 \text { nella forma a lemma), sostenuto dagli ipo- } \\
\text { coristici Ghita (2) e Chita (1) }\end{array}$ & 8 & $5 ; 2 ; 1$ \\
\hline Bartolomea, Bartola & 8 & $7 ; 1$ \\
\hline Francesca; Checca & 7 & $6 ; 1 ?$ \\
\hline Antonia & 5 & 5 \\
\hline Beatrice, Bice & 4 & $2 ; 2$ \\
\hline
\end{tabular}

Questi dati permettono di ricostruire il quadro onomastico dell'epoca, associandolo a personaggi concreti, esponenti della borghesia mercantile, eventualmente utilizzabili per confronti con altri repertori femminili dello stesso periodo.

Osservazioni linguistiche

Propongo ora alcuni esempi di confronti e considerazioni linguistiche che è possibile effettuare a partire dalla grande ricchezza di varianti grafiche e fonetiche fornite dal corpus.

Possiamo confrontare, ad esempio, le diverse rese grafiche del fonema nasale palatale $/ \mathrm{n} /$, ritrovando la consueta varietà che caratterizza la scripta toscana ${ }^{23}$ : a fianco a $g n$ troviamo anche gni e $n g n$ :

Tabella 8

\begin{tabular}{|c|c|}
\hline Forma & Conteggio di forma \\
\hline agnola & 1 \\
\hline agnola di lotto & 1 \\
\hline angniola & 1 \\
\hline angnola & 2 \\
\hline ghramignia & 1 \\
\hline tingna & 1 \\
\hline
\end{tabular}

22 Cfr. Tecchini Caterina (Tina) di Niccolò dell'Ammannato.

23 Castellani 1991: 27-29. 
Un'altra particolarità della scripta toscana è ravvisabile nella resa grafica dell'occlusiva velare sorda $/ \mathrm{k} /$, al cui proposito Castellani afferma, portando ad esempio il Libro del dare e dell'avere di Lapo Riccomanni (1281-1297), che l'autore tende a separare le occlusive velari dalle affricate palatali usando per le prime, di preferenza ma non unicamente, il digramma $c h$, sia dinanzi a $i$ ed $e$, sia dinanzi ad $a, o, u^{24}$, come accade nei nomi del corpus:

Tabella 9

\begin{tabular}{|c|c|}
\hline Forma & Conteggio di forma \\
\hline chara & 1 \\
\hline chatarina & 3 \\
\hline chaterina & 5 \\
\hline chaterina d'andrea & 1 \\
\hline chaterina d'aringho & 1 \\
\hline chaterina di barnabò & 1 \\
\hline chaterina di bernabò & 1 \\
\hline chaterina di nicholozo & 1 \\
\hline checcha & 1 \\
\hline domenicha & 1 \\
\hline fancescha & 1 \\
\hline fraciescha & 1 \\
\hline francescha & 3 \\
\hline franciescha & 3 \\
\hline franciescha di franciescho & 1 \\
\hline franciescha di gusto & 1 \\
\hline Lucha & 1 \\
\hline
\end{tabular}

\section{CONCLUSIONI}

I corpora di testi medievali in italiano reperibili in rete possono effettivamente fornire materiale interessante per la ricerca antroponimica, ma non tutti sono adatti ad una ricerca puntuale: il corpus TLIO non permette di distinguere efficacemente individui reali da personaggi letterari, a causa dell'assenza di una lemmatizzazione omogenea e di mappe distintive.

DATINI, invece, grazie alla presenza di solo materiale documentario, permette l'estrazione dei nomi di soli individui reali, rendendo così efficace la ricerca antro-

\footnotetext{
${ }^{24}$ Ibidem: 30 .
} 
ponimica, pur con tutti i limiti del caso e le numerose operazioni di aggiustamento che lo studioso di onomastica deve mettere in atto per poter lavorare adeguatamente sui dati estratti.

\section{BIBLIOGRAFIA}

Amenta N. 1753: "Il forca", commedia di Niccolò Amenta, avvocato napoletano, Stamperia de Muzj, Napoli.

Artale E. 2006: Mercanti medievali in Internet: le lettere dell'archivio Datini in GattoWeb, [in:] Prospettive nello studio del lessico italiano. IX Congresso SILFI, Firenze, 14-17 giugno 2006, Firenze University Press, Firenze.

Brattö O. 1953: Studi di antroponimia fiorentina. Il Libro di Montaperti (An. MCCLX), Elanders Boktryckeri Aktiebolag, Göteborg.

Brattö O. 1955: Nuovi studi di antroponimia fiorentina. I nomi meno frequenti del Libro di Montaperti (An. MCCLX), Almquist \& Wiksell, Stockholm.

Castellani A. 1991: Problèmes concernant la notion de scripta et problèmes concernant le rapport entre graphèmes et phonèmes, [in:] Actes Trèves 1991, pp. 695-699 [rist. col titolo Grafemi e fonemi: esempi medievali, [in:] Id., Nuovi saggi di linguistica e filologia italiana e romanza (1976-2004), a cura di V. Della Valle et al., Roma, Salerno Editrice, 2010].

Mosti R. 2010: Italiano antico e italiano moderno: notizie dal TLIO, Giornata di studi del Consiglio Nazionale delle Ricerche "Lo stato della lingua. Il CNR e l'italiano nel terzo millennio", Roma.

\section{SITOGRAFIA}

Archivio di Stato di Prato, http://datini.archiviodistato.prato.it/ Corpus TLIO, http://tlioweb.ovi.cnr.it//HelpCorpora/Infotlio.html Corpus DATINI, http://aspweb.ovi.cnr.it/(S(mwqipyavxrpipynbklbatfzj))/HelpCorpora/Infoasp.html GATTOWEB, http://gattoweb.ovi.cnr.it

OVI, http://www.ovi.cnr.it

\section{FEMININE NAMES IN ONLINE CORPORA OF ITALIAN MEDIEVAL DOCUMENTS}

\section{SUMMARY}

Corpora and network tools which provide access to Italian medieval documentation are nowadays accessible online. The current research is now oriented mainly towards lexical and linguistic aspects. My purpose is to investigate the use of these resources for an anthroponimical study, to verify the accessibility of the different network tools and, finally, to consider which kind of results can be obtained and also the possibility to extract data and materials, in order to distinguish onomastic types: placenames and anthroponyms (male and female names, first name, second name, nickname, etc.). My research is particularly focused on feminine names found in Corpus TLIO and Corpus DATINI.

K e y w ord s: TLIO, DATINI, anthroponymic corpora, medieval names, variants 